Editorial

\title{
Let's Collaborate More and Together Take India to the Next Level!
}

\author{
Sanjeeva Kalva ${ }^{1}$ Shyamkumar N. Keshava² \\ ${ }^{1}$ Division of Interventional Radiology, Department of \\ Radiology, Massachusetts General Hospital, Boston, \\ Massachusetts, United States \\ ${ }^{2}$ Department of Interventional Radiology, Division of Clinical \\ Radiology, Christian Medical College, Vellore, Tamil Nadu, India \\ J Clin Interv Radiol ISVIR 2019;3:149-150
}

With the growth of subspecialization in every field of medicine, there are often new therapeutic options for the same disease from different subspecialties. For example, a $3.5 \mathrm{~cm}$ hepatocellular carcinoma in the left lobe of the liver in a 72-year-old with Child-Pugh (CP) A cirrhosis and portal hypertension with an Eastern Cooperative Oncology Group performance score of 1 can be treated with a variety of therapeutic options-resection, thermal ablation, bland embolization, chemoembolization, radioembolization, stereotactic body radiation therapy, or some combination of these and medical therapy. The treatment would most likely be governed by local expertise, availability of treatment, affordability, and patient preference. Many would believe that a randomized study would make it easier to decide on therapies. Strict selection criteria in clinical studies, however, limits the generalization of the outcomes in patients outside of the selection criteria. Sorafenib, for example, is approved for treatment of hepatocellular carcinoma as it was proven to improve survival outcomes (by 2.8 months) in CP A patients (with additional specific criteria for platelet count and hepatic and renal function) compared with placebo. ${ }^{1}$ It is unknown whether Sorafenib would have similar effect in CP B patients. Systematic reviews, meta-analysis, network meta-analysis, propensity score matching for statistical analysis of observational data are some of the methods applied to assess the comparative effectiveness of various treatments when direct randomized data are not available. It is important to note that the technology and drugs evolve in continuum so frequent analysis of data are required to understand the comparative outcomes of the treatment options.

\section{Collaborative Care}

As such, a collaborative clinical decision model that involves the pertinent medical specialists would improve patient outcomes. ${ }^{2}$ Currently, many institutions in India and abroad

Address for correspondence Shyamkumar N. Keshava, MBBS, DMRD, DNB, FRCR, FRANZCR, Department of Interventional Radiology, Division of Clinical Radiology, Christian Medical College, Vellore 632004, Tamil Nadu, India (e-mail: aparna_shyam@cmcvellore.ac.in).

routinely practice "multidisciplinary conferences and clinics" to treat patients with cancer. This can be extended to other diseases as well. The prime targets suited for interventional radiology practice include uterine fibroids, aortic aneurysms, vascular diseases, stroke, arteriovenous malformations, benign prostatic hyperplasia and palliative therapy for pain. One could go further in developing "Centers of Excellence" with this model-examples include a fibroid center, aortic disease center, etc. Collaboration can be extended to practice patterns. Many interventional radiology (IR) physicians in India practice as individual consultants or are a part of radiology at a hospital or academic institution. One can explore models based on disease, for example, 'Liver Cancer' practice group or 'Vascular Disease' practice group. Such models of practice require substantial commitment and trust among the subspecialty physicians but would improve the overall patient care by providing 'one stop shop' for treatment of a particular problem often requiring multiple visits to multiple specialists. These collaborative practices would allow improved visibility of interventional radiologists and will showcase the value of interventional radiology in daily clinical practice. Additionally, the improved patient outcomes will benefit all participating specialties.

\section{Learning Together}

A collaborative learning platform is now part of many national society meetings. The Indian Society of Vascular and Interventional Radiology (ISVIR) has made it a priority to include physicians from other specialties at their annual meetings to discuss recent developments in therapeutic options for our patients. Case-based panel discussions are one of the ways to review "personalized medicine" choices. Similarly, collaborative learning in regular didactic sessions may be applied by including other specialists in the teaching curriculum of postgraduate training. In this world of information technology,

C2019 by Indian Society of Vascular and Interventional Radiology

\section{License terms \\ $($ () (1) $\Theta \circledast$}


'disease specific groups' of physicians across the world can discuss the evolving data and support choices for patients using web conferences and other messaging applications.

\section{Developing Guidelines}

Evidence-based clinical practice guidelines are useful for guiding physicians on effective treatment options for specific diseases. The Society of Interventional Radiology (SIR), American College of Radiology (ACR), Cardiovascular Interventional Society of Europe (CIRSE), and other societies routinely publish such clinical practice guidelines for IR. It is time for ISVIR to focus on developing similar guidelines for Indian patients. This can be achieved by improved collaboration across the SIR, CIRSE, and other medical societies and working with their team of researchers on clinical practice guidelines. While generalized guidelines across the world would be highly beneficial, it is important to take into consideration the variations in patient populations, patient comorbidities, local expertise, availability of treatments, cost, and patient preferences and develop nation-specific guidelines for India. These would immensely help our patients and physicians.

\section{Building a Pipeline}

The viability of IR in India is highly governed by the availability of interventional radiologists, decreasing the cost of disposables used in IR therapies, and federal/state regulations supporting the use of interventional therapies under various health insurance schemes. These require more collaboration at the grass roots level. Academic interventional radiologists must consider active participation in teaching medical students about IR and forming 'interest groups' to explore more options for mentoring and clinical rotations in IR. Given that the opportunities for training in IR are nonuniform across India, a standardized curriculum, accreditation of training programs and certification should be considered by the ISVIR. For a greater impact and unbiased evaluation, the ISVIR could partner with global IR societies (such as the SIR or CIRSE) in creating a process for accreditation and certification of IR training in India. Similarly, exchange programs with IR training centers across the world would benefit interventional radiologists in India. One such program is the ISVIR-SIR exchange program where a few selected interventional radiologists from India attend the annual meeting of the SIR and spend a month observing IR practices at major universities in the USA. India, as a nation, is known for the knowledge, skills, and endurance of young engineering scientists from the Indian Institute of Technology (IIT). The ISVIR should look into collaborating with IITs and other engineering institutions to locally develop new innovative ways of reducing the cost of disposables used in IR. There are opportunities to engage Western and East Asian manufacturers to set up local production centers to reduce the cost of IR disposables. Similarly, local manufacturers in India could be supported with preferential usage and engaged in research collaboration with IR centers for new product development. Recent support from the federal authorities through "Make in India" and "small business entrepreneurship" could help jumpstart such endeavors. Such collaborations can be extended and explored for all the South Asian Association for Regional Cooperation (SAARC) nations and it is possible that India could be at the leading edge of the world in providing IR therapies at a fraction of the cost in developed nations. To make IR therapies available to the poor, it is important for the ISVIR and leaders in IR to work with the authorities to make these therapies reimbursable or covered under state and federal insurance schemes.

Let us meet at Goa next year for our annual meeting and discuss more about collaborating!

\section{Conflict of Interest}

None declared

\section{References}

1 Llovet JM, Ricci S, Mazzaferro V, et al; SHARP Investigators Study Group. Sorafenib in advanced hepatocellular carcinoma. N Engl J Med 2008;359(4):378-390 10.1056/NEJMoa0708857

2 Yopp AC, Mansour JC, Beg MS, et al. Establishment of a multidisciplinary hepatocellularcarcinoma clinic is associated with improved clinical outcome. Ann Surg Oncol 2014;21(4):1287-1295 10.1245/s10434-013-3413-8 